\title{
COMMISSION 32. (SELECTED AREAS.)
}

President: Prof. P. J. van Rhijn.

SeCRETARY: Dr C. H. Hins.

The draft report was adopted with one or two minor alterations.

The Commission discussed the points mentioned in section I 2 in the report, "desiderata for future work."

I. Dr Shapley promised to consider the possibility of using the Bruce telescope for the Durchmusterung of the southern areas, going down to magnitude I8.5 approximately.

3. The President asked Dr Jackson's opinion about the possibility of observing the meridian positions of the standard stars of the southern areas from $-30^{\circ}$ downward at the Cape observatory.

Dr Jackson's objection to such work was on account of the faintness of these standard stars. The whole southern sky.from $-30^{\circ}$ to the south pole will be repeated photographically with the wide angle lens.

4. Dr Adams promised to extend the Mount Wilson programme of radial velocities observations to the areas of $-30^{\circ}$.

As to the radial velocities of stars fainter than 9.0 m., Dr Adams promised collaboration with the spectrograph used at present by $\mathrm{Mr}$ Joy in the determination of radial velocities of Cepheids, after these observations had been finished.

Dr Shapley gave an account of Dr Bok's work on radial velocities by means of an objective prism. The work is still in an experimental state; it is expected that the radial velocities of tenth magnitude stars can be found with a p.e. of $7 \mathrm{~km} . / \mathrm{sec}$.

Dr Shapley will consider the possibility of including the selected areas in the working programme.

As to the absorption of light in space, Dr Shapley remarked on the desirability of examining the plates eventually to be taken with the Bruce telescope (see r) for the distribution of extra-galactic nebulae, this distribution giving indications about irregularities in the absorption of light in space.

\section{COMMISSION 33. (STELLAR STATISTICS.)}

President: Prof. B. Lindblad.

SECRETARY: Dr C. Schalén.

A recommendation to the Union concerning the construction of certain tables was accepted.

A discussion was opened on the subject of collaboration in the study of open clusters. An application to the General Assembly was passed for a recommendation as follows: "That the Union call attention to the important problems connected with the open clusters and determinations of proper motions, radial velocities, magnitudes and spectra along the lines already inaugurated at several observatories."

It was agreed that Dr Mineur should approach the observatories of the Carte $d u$ Ciel on the subject of photographing the open clusters for the future determinations of proper motions. 
DrLuyten's suggestion concerning catalogues of orbits of visual and spectroscopic binaries was discussed. The following recommendation to the General Assembly was passed: "That the Union recommend that an observatory publish at frequent intervals complete catalogues of all known orbits of visual and spectroscopic binaries."

Dr Parvulesco recommended the use of a certain standard system of projection for studying the galactic distribution of celestial objects.

It was agreed to recommend to the General Assembly the adoption of the galactic pole $\alpha=\mathrm{r} 2^{\mathrm{h}} 4 \mathrm{O}^{\mathrm{m}}, \delta=+28^{\circ}$ ( $\mathrm{rgOO}$ ) as a standard for computing galactic co-ordinates.

\title{
COMMISSION 34. (SOLAR PARALLAX.)
}

\author{
President: Di Spencer Jones. \\ Secretary: Prof. F. Slocum.
}

The President first summarized the present position of the work of determining the solar parallax as given in the Report.

In connection with the determination of the positions of the reference stars it was stated that within the next six months or so the reductions of all the series of photographs will be completed and it will then be possible to co-ordinate the results and publish a definitive list of secondary comparison stars. At the same time it has become apparent that there are appreciable errors in the positions based on the meridian observations of some of the primary stars given in Prof. Kopff's catalogue. By co-ordinating the measures of the several series of path plates it will be possible to derive corrections to the positions of the Kopff stars, which will still keep them on the system adopted by Prof. Kopff, but will improve their homogeneity.

The second portion of the work of the Commission concerns the ephemeris of Eros. This work is being carried on by Prof. Witt with the aid of Dr von Schelling. Supplementing the statements in the Report, Prof. Witt states that he finds a regular run in the differences between calculation and observation, and he thinks it is, therefore, necessary to include a correction to the mass of the earth as an additional unknown in the solution. The demi-definitive ephemeris, which has been published in the Astronomische Abhandlungen, is sufficiently accurate for the purpose of deriving the solar parallax by the decimal method but is scarcely of the accuracy needed for the derivation of the mass of the Moon. It is desirable that the ephemeris should be improved by working backwards and connecting on to previous observations by applying general perturbations. Prof. Witt is working along this line and hopes that funds will be available for the continued employment of Dr von Schelling as computer.

From the large number of observations taken around the opposition of I93I, the solar parallax, by the diurnal method or by observations made almost simultaneously at different stations, will be derived with a very small probable error, but it is possible that systematic errors, due to differences in colour of Eros and the comparison stars, etc., may be appreciably larger than the internal errors. The best determination of the solar parallax may ultimately come from the gravitational method along the lines developed by Prof. Noteboom. Therefore the continuance of the investigations of Prof. Witt to obtain the most accurate determination of the orbit of Eros is a piece of work that should be supported by this Commission. 